\title{
Prescription en ambulatoire de médicaments «hors LS» et/ou soumis à autorisation préalable de l'assureur
}

\author{
Les auteurs rappellent la procédure d'enregistrement des médicaments en Suisse, \\ puis présentent les exceptions jurisprudentielles et légales et les démarches à entre- \\ prendre pour la prescription et le remboursement de médicaments dans ces condi- \\ tions particulières.
}

Mirela Cacia,

Jeanne-Pascale Simon ${ }^{b}$, Jean-Blaise Wasserfallen ${ }^{c}$

a Directrice médicale adjointe Centre hospitalier universitaire vaudois CHUV

b Juriste, Unité des affaires juridiques CHUV

c Professeur titulaire UNIL, MPP, Directeur médical CHUV

\author{
Correspondance: \\ Mirela Caci \\ Centre hospitalier universitaire \\ vaudois CHUV \\ Bugnon 21 \\ CH-1011 Lausanne \\ Tél. 0213141435 \\ Fax 0213141818 \\ mirela.caci[at]chuv.ch
}

\section{Introduction}

Certains médicaments ne sont pas admis à la vente en Suisse ou s'ils le sont, ne sont pas à la charge de l'assurance obligatoire de soins (OPAS) [1]. Pourtant, leur utilisation peut s'avérer utile, voire indispensable, pour traiter certains patients. Cette prescription particulière est possible, mais nécessite de respecter un certain nombre de règles, notamment d'informer complètement le patient de la situation et de ses conséquences, et de réaliser quelques démarches administratives auprès des autorités sanitaires et de la caisse-maladie du patient.

Cet article rappelle la procédure d'enregistrement des médicaments en Suisse, puis présente les exceptions légales et les démarches à entreprendre pour la prescription et le remboursement de médicaments dans ces conditions particulières.

\section{L'enregistrement des médicaments en Suisse}

\section{Swissmedic et l'Autorisation de Mise} sur le Marché (AMM)

Pour accompagner sa demande d'enregistrement auprès des autorités d'enregistrement suisses (Swissmedic), le fabriquant doit faire état de trois études randomisées contrôlées montrant l'efficacité de son médicament par rapport à un placebo. Une fois l'efficacité et la sécurité du médicament établies, une AMM lui est accordée pour une indication précise (articles 10 et 11 de la Loi fédérale sur les produits thérapeutiques, LPTh) [2]. Cette AMM spécifie l'indication pour laquelle le médicament est autorisé, sa posologie d'administration, la durée du traitement, les patients concernés et éventuellement l'ordre d'utilisation des traitements. L'ensemble de ces données figure dans l'information professionnelle, sous forme d'une notice d'emploi qui accompagne l'emballage du médicament et qui figure également dans le Compendium suisse des médicaments.

\section{Résumé}

L'utilisation de certains médicaments non admis à la vente en Suisse ou non à la charge de l'assurance obligatoire de soins (AOS) est possible sous certaines conditions définies dans les exceptions jurisprudentielles inscrites dans l'ordonnance sur l'assurance-maladie (OAMal). En plus des informations usuelles sur le médicament et les démarches à entreprendre auprès de l'assureur, le médecin doit informer son patient du risque de refus de remboursement de ce dernier, faute de quoi sa responsabilité peut être engagée et les coûts du médicament peuvent se retrouver à sa charge. Tandis que certains Ofacteurs semblent tendre à faciliter l'accès à ce genre de traitement, d'autres contribuent clairement à le rendre plus difficile.

Une prescription hors AMM est admissible sur le plan du droit civil et pénal pour autant que l'intérêt du patient le justifie et qu'il ait donné son consentement éclairé. Le patient s'expose à des conséquences financières, et le médecin à des conséquences juridiques potentielles au cas où des complications survenaient lors du traitement.

\section{L'Office Fédéral de la Santé Publique (OFSP) et la Liste des Spécialités (LS)}

Une fois l'AMM obtenue, le fabriquant de médicaments a la possibilité de déposer un dossier auprès de l'OFSP pour inscrire son médicament sur la Liste des Spécialités (LS) [3] qui sont ainsi à charge de l'assurance obligatoire de soins (AOS). Pour ce faire, il doit 
démontrer l'efficacité du traitement, son caractère approprié et son économicité. Les deux premiers points sont en principe repris de l'AMM, et seul le troisième est spécifiquement étudié.

Cet examen s'effectue par comparaison géographique avec les prix du même médicament dans un panier de pays européens (Allemagne, Danemark, Angleterre, Pays-Bas, France, Autriche) et par comparaison avec d'autres médicaments qui constituent des alternatives thérapeutiques et qui sont déjà inscrits dans la LS. La comparaison porte sur le coût par jour et le coût par traitement. Pour une molécule innovante, une prime à l'innovation (10 à 20\%) peut être accordée, de même qu'un supplément pour le traitement d'une maladie grave. Le prix ainsi déterminé est obligatoire.

\section{Cette prescription particulière nécessite de respecter} un certain nombre de règles, notamment d'informer complètement le patient de la situation et de ses conséquences.

Pour les médicaments non inscrits dans la LS, y compris pour les médicaments hospitaliers, le prix est libre.

La LS est une liste positive, c'est-à-dire complète, exhaustive et contraignante pour la question de la prise en charge par l'AOS. Elle précise la forme galénique, le dosage et la taille de l'emballage admis à l'AOS. Elle peut également définir des limitations en ce qui concerne l'indication thérapeutique, le dosage, la durée du traitement, l'ordre des traitements, la gravité de l'état de santé, les modalités de facturation, les caractéristiques des médecins autorisés à le prescrire, la surveillance médicale exigée et la nécessité d'obtenir au préalable l'accord du médecin-conseil.

Il en découle que les caisses-maladie ont l'interdiction de rembourser des médicaments hors liste, sauf les exceptions traduites dans les articles 71a et $71 \mathrm{~b}$ de l'Ordonnance sur l'assurance-maladie (OAMal) [4], et qu'elles peuvent refuser de rembourser un traitement en cas de non-respect des limitations (prescriptions hors étiquette). En résumé et sauf exception, seuls les médicaments inscrits sur la LS - et dans les limites qui y sont indiquées - sont remboursés par l'AOS.

\section{Les exceptions}

Elles sont au nombre de 4, les deux premières relevant de la jurisprudence du Tribunal fédéral, la troisième et la quatrième des instructions de l'OFSP concernant la Liste de spécialités. Ces exceptions ont, depuis le $1^{\text {er }}$ mars 2011, été inscrites dans les articles 71a et 71b de l'OAMal.

\section{Le complexe thérapeutique}

Un médicament non-LS peut être remboursé s'il constitue un accessoire indispensable à un traitement admis à l'AOS. Il doit alors remplir trois conditions: un lien étroit avec le traitement admis à l'AOS, constituer un accessoire ou une étape préparatoire indispensable au traitement AOS et exister en l'absence d'autres mesures tout aussi appropriées. Un exemple de cette nature est l'utilisation du Neupogen en tant que mesure préparatoire indispensable à la transplantation du foie qui doit être prise en charge par la caisse-maladie [5].

\section{La nécessité thérapeutique}

Dans cette situation, le médicament en question doit constituer un bénéfice thérapeutique élevé pour un patient dont la santé est gravement menacée. Il doit alors remplir 4 conditions: la maladie non traitée mène à la mort ou à une atteinte grave et chronique à la santé, aucune alternative efficace n'existe dans l'AOS, le médicament possède une utilité thérapeutique importante (curative ou palliative), et des preuves scientifiques existent [6]. Un exemple récemment jugé dans cette catégorie est le remboursement du Myozyme ${ }^{\circledast}$ pour le traitement de la maladie de Pompe dans sa forme adulte [7].

\section{Le médicament orphelin}

Dans ce cas de figure, le médicament doit pouvoir traiter une population réduite (prévalence $\leq 5$ patients pour 10000 personnes), affectée d'une maladie grave. Il doit également remplir les conditions suivantes: la maladie non traitée mène à la mort ou à une atteinte grave et chronique à la santé, aucune alternative n'existe dans l'AOS, des preuves scientifiques existent et l'accord du médecin-conseil a été obtenu. Un exemple de cette catégorie est représenté par le traitement de maladies métaboliques rares.

\section{Usage compassionnel}

Il s'agit de la distribution ou de la remise, pour une durée limitée, de médicaments qui ne sont pas autorisés à être mis sur le marché en Suisse, et destinés à traiter des maladies autrement mortelles.

Pour pouvoir être administré à ce titre, le médicament doit remplir les conditions suivantes: le patient doit être nommément identifié; il doit souffrir d'une maladie grave qui met sa vie en danger ou est responsable d'une invalidité; une grande utilité thérapeutique est attendue; il n'existe pas d'alternative thérapeutique; il s'agit d'une intervention en urgence ou d'une dernière possibilité thérapeutique; le médicament est déjà autorisé ou en procédure d'admission dans un pays tiers; il existe des études cliniques ayant apporté des preuves scientifiques solides et l'accord du médecin-conseil a été obtenu. La demande de prescription d'un médicament à usage compassionnel est effectuée auprès de Swissmedic. 
Dans les quatre situations ci-dessus (1.-4.), si les critères des art. 71a et b OAMal sont remplis, le médicament devra être remboursé par l'assureur qui en fixe le prix.

\section{Démarches à entreprendre auprès de l'assureur-maladie}

Après avoir décidé avec son patient de l'attitude thérapeutique, le médecin doit l'informer sur les bénéfices et les risques associés au traitement et les démarches à initier auprès de l'assureur pour obtenir une décision de prise en charge. Il doit, en particulier, l'informer du risque de refus de remboursement par l'assureur, même d'une partie du prix du médicament, auquel cas les frais de ce traitement seraient à sa charge. En l'absence d'une telle information, la responsabilité du médecin, respectivement de l'institution qui l'emploie, peut être engagée et les coûts du médicament être à sa charge.

Avant d'initier le traitement, le médecin demande la prise en charge du médicament au médecin-conseil de l'assureur et ainsi une garantie spéciale de l'assureur en respectant les conditions des articles 71a ou 71b OAMal (cf. chiffres 1-4 ci-dessus).

Si l'assureur accepte la prise en charge et la confirme par écrit, le traitement peut être administré aux conditions fixées par le médecin-conseil de l'assureur.

Si l'assureur refuse la prise en charge, une approche pragmatique pourrait être de lui opposer une

\section{Si l'assureur persiste dans son refus, le médicament peut tout de même être administré au patient, pour autant qu'il le paie.}

argumentation juridique et d'adresser une nouvelle demande au médecin-conseil de l'assureur.

En cas de nouveau refus, si le patient et/ou son médecin le souhaitent, un recours auprès d'un tribunal ou l'intervention d'organismes externes tels que l'Ombudsman de l'assurance-maladie, l'Office fédéral de la santé publique, l'Association Suisse des Assurés, l'Organisation Suisse des Patients, etc., sont possibles.

Finalement, si l'assureur persiste dans son refus, le médicament peut tout de même être administré au patient, pour autant qu'il le paie ou trouve un financement lui-même.

Ce n'est que si le patient accepte le risque de refus de prise en charge par l'assureur, que le médicament peut être administré avant la fin de la procédure menée auprès du médecin-conseil de l'assureur.

A noter que dans le laps de temps nécessaire à l'obtention d'une réponse de l'assureur, le médecin doit réévaluer les options thérapeutiques à disposi- tion du patient selon sa situation et les réponses de son assureur.

\section{Discussion}

L'accès d'un patient à un traitement médicamenteux «hors LS» et/ou soumis à autorisation préalable de l'assureur peut s'avérer être une démarche complexe, longue, et sans garantie de succès.

Trois facteurs peuvent être considérés comme favorables et susceptibles d'améliorer la situation à l'avenir:

- D'une part, la récente introduction des exceptions jurisprudentielles dans l'OAMal (art. 71a et b) qui a formalisé les conditions de prise en charge de certains médicaments et apporté ainsi une clarification nécessaire.

- D'autre part, une modification de l'ordonnance sur les médicaments survenue en juillet 2010 prévoit que si un médicament ou un procédé a déjà été autorisé à la mise sur le marché dans un pays ayant institué un contrôle des médicaments équivalent à celui qui existe en Suisse, les résultats des essais effectués en vue de l'octroi de l'autorisation dans ce pays sont pris en considération. Ceci devrait notablement raccourcir la procédure d'enregistrement.

- Par ailleurs, le mouvement politique existant actuellement en faveur des maladies rares ainsi que la sensibilisation de l'opinion publique par quelques exemples de difficultés de remboursement de médicaments dans des situations particulièrement sensibles sont susceptibles de jouer un rôle favorable à l'acceptation du remboursement par les assureurs et notamment leur médecin-conseil.

Toutefois, d'autres éléments constituent plutôt des menaces sur l'accès aux médicaments:

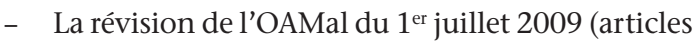
65 et suivants) qui instaure un contrôle par l'OFSP des nouvelles indications autorisées par Swissmedic, bien que l'admission automatique reste la règle en pratique.

- D'autre part, une modification des exceptions jurisprudentielles a récemment été notée sous la forme de l'introduction de la notion de rapport coût-bénéfice acceptable. Cela a été le cas en 2008 avec un arrêt du Tribunal fédéral sur un traitement par Herceptin [8], pour lequel un seuil maximum de 50000 Euro par année a été évoqué, ou plus récemment avec l'arrêt sur le Myozyme où un seuil de 100000 francs a également été mentionné [7].

Les menaces les plus sérieuses sont toutefois à rechercher dans les modifications d'attitude des caissesmaladie. D'une part, les compétences des médecinsconseils sont hétérogènes selon les caisses, de sorte que des attitudes divergentes face à des situations semblables peuvent être notées entre les différentes caisses-maladie. L'aptitude à gérer ce type de de- 


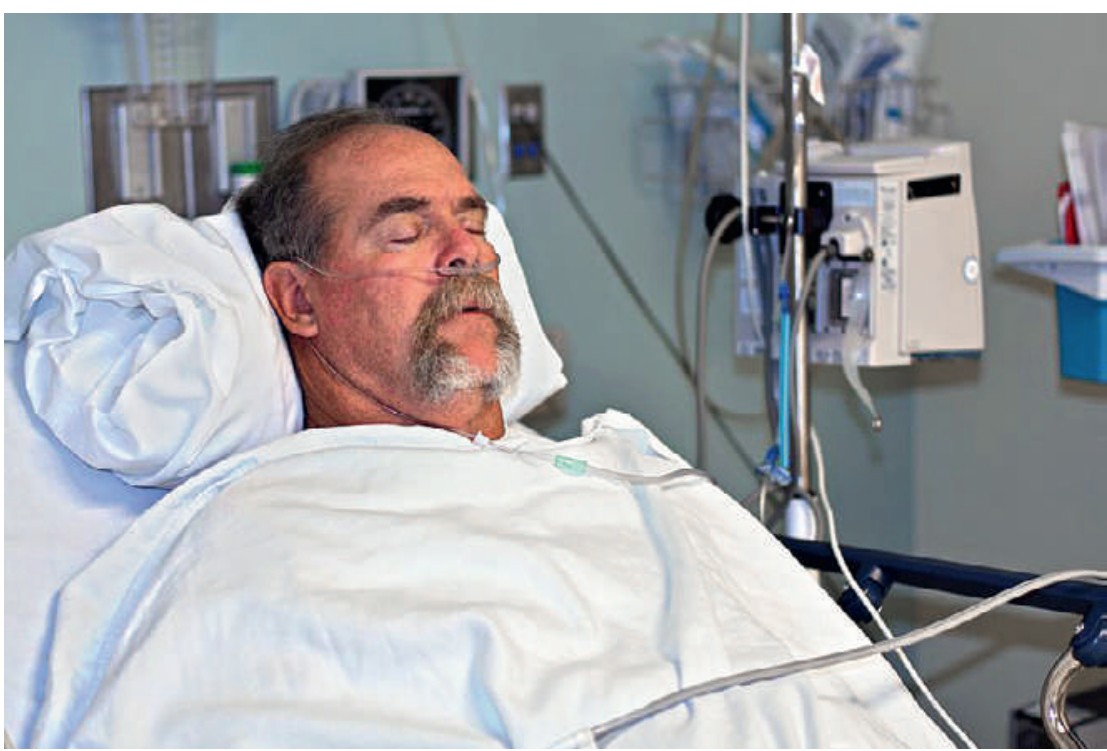

Un médicament «hors LS» doit remplir plusieurs conditions, entre autres que la maladie non traitée mène à la mort ou à une atteinte grave et chronique à la santé et aucune alternative efficace n'existe dans l'AOS. chroniques qui nécessitent des médicaments onéreux.

Au total, l'évolution de ce sujet est difficile à prévoir. Si un certain nombre de facteurs positifs existent, plusieurs menaces potentielles sont également présentes, de sorte qu'une vigilance du corps médical est indispensable pour éviter les risques de dérapages préjudiciables au patient et rendre attentifs le monde politique et les instances de surveillance concernées.

La mise à disposition d'une information détaillée au médecin sur la manière de demander la prise en charge d'un médicament «hors LS» et/ou soumis à l'autorisation préalable du médecin-conseil en est un exemple. Dans ce domaine, une collaboration avec des juristes spécialisés et des organisations de patients est souhaitable pour maximaliser les chances de succès.

\section{Références}

1 Ordonnance du DFI du 29 septembre 1995 sur les prestations dans l'assurance obligatoire des soins en cas de maladie (Ordonnance sur les prestations de l'assurance des soins, OPAS) (www.admin.ch/ch/f/rs/ c832_112_31.html. Accès le 18.05.2012).

mandes qui concernent le plus souvent des situations médicales très pointues diverge également selon l'assureur-maladie. Alors que la plupart des caisses procèdent de manière correcte au contrôle des conditions de prise en charge des médicaments hors LS ou soumis à une autorisation préalable, certains assureurs abusent de leur position en réitérant les refus sans même apporter d'arguments. Le patient doit alors décider, d'aller jusqu'au bout de la procédure envers son assureur, d'y renoncer et supporter les coûts du traitement ou constater qu'il ne peut bénéficier de ce traitement de choix et trouver, avec l'aide de son médecin, la moins mauvaise alternative thérapeutique prise en charge par l'AOS. Pour faire face à cette situation, quelques initiatives récentes méritent d'être signalées: un modèle d'évaluation coût/utilité incorporant qualité et durée de vie, proposé par J. Nadig [9], et le modèle de notation utilisé par l'assureur Helsana [10]. Reste à voir si ces initiatives sont acceptées par l'ensemble des parties impliquées.

Par ailleurs, la politique des caisses-maladie de passer du tiers payant au tiers garant constitue un obstacle économique difficilement contournable par les patients, qui doivent être traités par des médicaments extrêmement chers et se retrouvent dans l'impossibilité d'avancer les sommes nécessaires à leur traitement en attendant un remboursement par l'assureur. Finalement, la campagne permanente des assureurs pour la suppression de l'obligation de contracter leur permettrait en cas de succès d'exclure un certain nombre de médecins, dont en particulier ceux qui s'occupent du traitement des maladies rares ou
2 Loi fédérale du 15 décembre 2000 sur les médicaments et les dispositifs médicaux (loi sur les produits thérapeutiques, LPTh) (www.admin.ch/ch/f/rs/ c812_21.html. Accès le 18.5.2012).

3 Office fédéral de la santé publique. Liste des spécialités (www.bag.admin.ch/themen/krankenversicherung/ 00263/00264/00265/index.html?lang=fr. accès le 18.5.2012).

4 Ordonnance du DFI du 27 juin 1995 sur l'assurance maladie (OAMal) (www.admin.ch/ch/f/rs/8/832.102. fr.pdf. Accès le 18.5.2012).

5 Tribunal Fédéral. Arrêt K 991/1998 du 11 mai 1998, publié dans RAMA 4/1998, p. 302 (www.bag.admin.ch/ themen/krankenversicherung/02874/02876/index. html?lang=fr\&download=NHzLpZeg7t,lnp6I0NTU04 2l2Z6ln1ae2IZn4Z2qZpnO2Yuq2Z6gpJCFeIN9f2ym 162epYbg2c_JjKbNoKSn6A-- . Accès le 18.5.2012).

6 Tribunal Fédéral. Arrêt K 103/03 du 14 septembre 2004, publié aux ATF $130 \mathrm{~V}$ 532. (http://relevancy.bger.ch/ cgi-bin/JumpCGI?id=BGE-130-V-532\&lang=fr\&zoom= OUT\&system=clir. Accès 18.5.2012).

7 Tribunal Fédéral. Arrêt 9C_334/2010 du 23 novembre 2010, publié aux ATF 136 V 395. (http://jumpcgi.bger. ch/cgi-bin/JumpCGI?id=23.11.2010_9C_334/2010. Accès le 18.5.2012)

8 Tribunal Fédéral. Arrêt 9C_56/2008 du 6 Octobre 2008. (http://jumpcgi.bger.ch/cgi-bin/JumpCGI?id=06.10.2 008_9C_56/2008. Accès le 18.5.2012).

9 Nadig J. Evidenzbasierte Nutzenbewertung: ein Modell zur Vergütung des Off Label Use in der Onkologie. Bull Méd Suisses. 2012;93(14/15):552-5.

10 Seiler B, Fries R, Honegger HP. Evaluation du bénéfice des médicaments hors étiquette. Bull Méd Suisses. 2012;93(19):723-5. 